\title{
Symmetric Digraphs from Powers Modulo $n$
}

\author{
Guixin Deng $^{1 *}$, Pingzhi Yuan ${ }^{2}$ \\ ${ }^{1}$ School of Mathematics, Guangxi Teachers Education University, Nanning, China \\ ${ }^{2}$ School of Mathematics, South China Normal University, Guangzhou, China \\ E-mail: ${ }^{*}$ oldlao@163.com,mcsypz@mail.sysu.edu.cn
}

Received June 21, 2011; revised July 25, 2011; accepted August 5, 2011

\begin{abstract}
For each pair of positive integers $n$ and $k$, let $G(n, k)$ denote the digraph whose set of vertices is $H=\{0,1,2, \cdots$, $n-1\}$ and there is a directed edge from $a \in H$ to $b \in H$ if $a^{k} \equiv b(\bmod n)$. The digraph $G(n, k)$ is symmetric if its connected components can be partitioned into isomorphic pairs. In this paper we obtain all symmetric $G$ $(n, k)$.
\end{abstract}

Keywords: Congruence, Digraph, Component, Height, Cycle

\section{Introduction}

In [12], L. Szalay showed that $G(n, 2)$ is symmetric if $n \equiv 2(\bmod 4)$ or $n \equiv 4(\bmod 8)$. In [1], the authors proved that if $p$ is a Fermat prime, then $G\left(2^{r} p, 2\right)$ is not symmetric when $r=3$ or $r=5$, but it is symmetric when $k=4$. And the following theorem is part of Theorem 5.1 in [13].

Theorem 1.1 ([13] Theorem 5.1) Let $n=n_{1} n_{2}$, where $n_{1}>1, n_{2}>1$ and $\operatorname{gcd}\left(n_{1} n_{2}\right)=1$. Suppose that $n_{1}=2^{m}$, where $m \geq 1$. Then $G(n, k)$ is symmetric if one of the following conditions holds:

i) $m \leq 2, k \geq 2$, and $2^{m-1} \mid k$;

ii) $m \geq 3, k>2$, and $2^{m-2} \mid k$;

iii) $m=4$ and $k=2$

In this paper we prove that if $G(n, k)$ is symmetric, where $k \geq 2$ and $2^{\mathrm{m}} \| n$, then $m=5, k=4$ or $m, k$ satisfy one of the conditions of the above theorem.

The outline of this paper is as follows. In Section 3 we obtain all symmetric $G\left(2^{m}, k\right)$ by direct computation. In Section 4 we prove some properties about digraph products which will be useful in the proof of our main theorem. In Section 5 we state and prove the main theorem of the present paper.

\section{The Carmichael Lambda-Function}

Before proceeding further, we need to review some properties of the Carmichael lambda-function $\lambda(n)$.

Definition 2.1 Let $\mathrm{n}$ be a positive integer. Then the Carmichael-lambda-function $\lambda(n)$ is defined as follows:

$$
\begin{aligned}
& \lambda(1)=1 \\
& \lambda(2)=1 \\
& \lambda(4)=2, \\
& \lambda\left(2^{k}\right)=2^{k-2} \text { if } k \geq 3, \\
& \lambda\left(p^{k}\right)=(p-1) p^{k-1} \text { if } p \text { is an odd prime, } \\
& \lambda\left(\prod_{i=1}^{r} p_{i}^{e_{i}}\right)=\operatorname{lcm}\left[\lambda\left(p_{1}^{e_{1}}\right), \lambda\left(p_{2}^{e_{2}}\right), \cdots, \lambda\left(p_{r}^{e_{r}}\right)\right]
\end{aligned}
$$

where $p_{i}$ are distinc primes.

The following theorem generalizes the well-known Euler's theorem which says that $a^{\phi(n)} \equiv 1(\bmod n)$ if and only if $\operatorname{gcd}(a, n)=1$.

Theorem 2.1 (Camichael). Let $a, n \in \mathbb{N}$. Then $a^{\lambda(n)} \equiv$ $1(\bmod n)$ if and only if $\operatorname{gcd}(a, n)=1$. Moreover, there exists an integer $g$ such that $\operatorname{ord}_{n} g=\lambda(n)$, where $\operatorname{ord}_{n} g$ denotes the multiplicative order of $g$ modulo $n$.

For the proof see [5, p. 21]

\section{The Case $n=2^{m}$}

Let $G$ be a digraph and $a$ be a vertex in $G$. The indegree of $a$, denoted by ind $(a)$ is the number of directed edges coming to $a$, and the outdegree of $a$ is the number of edges leaving $a$. Particularly, let $\operatorname{ind}_{n}^{k}(a)$ denote the indegree of a vertex $a$ contained in $G(n, k)$.

There are two particular subdigraphs of $G(n, k)$. Let $G_{1}(n, k)$ be the induced subdigraph of $G(n, k)$ on the set of vertices which are coprime to $n$ and $G_{2}(n, k)$ be the induced subdigraph of $G(n, k)$ on the set of vertices which are not coprime with $n$. We observe that $G_{1}(n, k)$ 
and $G_{2}(n, k)$ are disjoint and that $G(n, k)=$ $G_{1}(n, k) \bigcup G_{2}(n, k)$, that is, no edges goes between $G_{2}(n, k)$ and $G_{2}(n, k)$.

It is clear that each component of $G(n, k)$ contains a unique cycle, since the component has only a finite number of vertices and each vertex has outdegree 1 . The following lemma tells us that every component contained in $G_{1}(n, k)$ is determined by its cycle length.

Lemma 3.1 ([13] Corollary 6.4) Let $t \geq 1$ be a fixed integer. Then any two components in $G_{1}(n, k)$ containing t-cycle are isomorphic.

Definition 3.1 We define a height function on the vertices and components of $G(n, k)$. Let $c$ be a vertex of $G(n, k)$, we define $h(c)$ to be the minimal nonnegative integer $i$ such that $c^{k^{i}}$ is congruent modulo $n$ to a cycle vertex in $G(n, k)$. And if $C$ is a component of $G(n, k)$, we define $h(C)=\sup _{c \in C} h(c)$.

The indegree and the height function play an important role in the structure of $G(n, k)$. We need the following results concerning the indegrees and heights.

Lemma 3.2 ([14]) Let $n=\prod_{i=1}^{r} p_{i}^{e_{i}}$ be the prime factorization of $n$. Let a be a vertex of positive indegree in $G_{1}(n, k)$. Then

$$
\operatorname{ind}_{n}^{k}(a)=\prod_{i=1}^{r} \operatorname{ind}_{p_{i}^{e_{i}}}^{k}(a)=\prod_{i=1}^{r} \delta_{i} \operatorname{gcd}\left(\lambda\left(p_{i}^{e_{i}}\right), k\right),
$$

where $\delta_{i}=2$ if $2 \mid k$ and $8 \mid p_{i}^{e_{i}}$, and $\delta_{i}=1$ otherwise.

Lemma 3.3 ([11] Theorem 3.2) Let $p$ be a prime. Let $a$ be a vertex of positive indegree in $G_{2}\left(p^{e}, k\right)$, and assume that $p^{l} \mid k$ and $a \neq 0$. Then $l=k$ for some positive integer $t$ and

$$
\operatorname{ind}_{p^{e}}^{k}(a)=\delta p^{(k-1) t} \operatorname{gcd}\left(\lambda\left(p^{e-l}\right), k\right)
$$

where $\delta=2$ if $p=2,2 \mid k$ and $e-l \geq 3$, and $\delta=1$ otherwise.

Lemma 3.4 ([13] Lemma 3.2) Let $p$ be a prime and e, $k$ be two positive integers. Then

$$
\operatorname{ind}_{p^{e}}^{k}(0)=p^{e-\left\lceil\frac{e}{k}\right\rceil} .
$$

Lemma 3.5 Let $p$ be a prime and $e \geq 2, k \geq 2$ be two positive integers. Let $h$ be the positive integer such that $k^{h-1}<e \leq k^{h}$. Then $h=h\left(G_{2}\left(p^{e}, k\right)\right)$.

Proof. It is clear that $p \in G_{2}\left(p^{e}, k\right)$ and $h(p)=$ $h\left(G_{2}\left(p^{e}, k\right)\right)$. And $p^{k^{i}} \equiv 0\left(\bmod p^{e}\right)$ if and only if $k^{i} \geq e$. This proves the Lemma.

Lemma 3.6 Let $p$ be a prime and $e, k \geq 2$ be two positive integers. Let $\lambda\left(p^{e}\right)=u v$ where $u$ is the maximal divisor of $\lambda\left(p^{e}\right)$ relatively prime to $k$. If $G$ is the component of $G\left(p^{e}, k\right)$ containing 1 , then

$$
h(C)=\min \left\{i: v \mid k^{i}\right\}
$$

Proof. Let $h=\min \left\{i: v \mid k^{i}\right\}$. Then there exists a divisor $d$ of $v$ such that $d$ is not a divisor of $k^{h-1}$. By Theorem 2.1 there exists a vertex $g \in G\left(p^{e}, k\right)$ such that $\operatorname{ord}_{p^{e}} g=u v$. Let $a \equiv g^{\frac{u v}{d}}\left(\bmod p^{e}\right)$. Then $\operatorname{ord}_{p^{e}} a$ $=d$ and $a^{k^{h-1}}$ is not congruent modulo $p^{e}$ to 1 , but $a^{k^{h}} \equiv 1\left(\bmod p^{e}\right)$. We have $h(C) \geq h(a)=h$ by the definition of height function.

Conversely if $a \in C$, then there exists $j \geq 1$ such that $a^{k^{j}} \equiv 1\left(\bmod p^{e}\right)$, then ord $_{p^{p^{a}}} a \mid k$. But ord ${ }_{p^{e}} a \mid u v$, hence $\operatorname{ord}_{p^{e}} a \mid v$. And $a^{k^{h}} \equiv 1\left(\bmod p^{e}\right)$. That is $h(C) \leq h$.

Lemma 3.6 is proved.

Now we can prove our first result.

Theorem 3.1 Let $k \geq 2, m \geq 1$ be two positive integers. Then $G\left(2^{m}, k\right)$ is symmetric if and only if one of the following conditions holds.
i) $m=1$;
ii) $m=2,2 \mid k$;
iii) $m=4, k=2$;
iv) $m=5, k=4$;
v) $m \geq 3,2^{m-2} \mid k, k \geq m$.

Proof. The case $m<3$ follows directly by simple computations, so we may assume that $m \geq 3$, thus $\lambda(2)=2^{m-2}$. We first suppose that $G\left(2^{m}, k\right)$ is symmetric. Let $C_{0}$ and $C_{1}$ be the components of $G\left(2^{m}, k\right)$ containing the vertex 0 and 1 , respectively. Then it is easy to see that $C_{0}$ is just $G_{2}\left(2^{m}, k\right)$. Since the cycle lengths of $C_{0}$ and $C_{1}$ are 1 , by the assumptions and Lemma 3.1 we must have $C_{0} \simeq C_{1}$, thus $h=h\left(C_{0}\right)=h\left(C_{1}\right)$.

If $h=1$, then $k \geq m$ and $\in \operatorname{gcd}\left(2^{m-2}, k\right)=$ ind (1) $=$ ind $(0)=2^{m-1}$, where $\in=1$ if $k$ is odd, and $\epsilon=2$ if $2 \mid k$. We must have $2^{m-2} \mid k$.

If $2 \nmid k$, then $C_{1}$ is a cycle, however $C_{0}$ is not a cycle. Hence we may assume that $2^{r}|| k, r \geq 1$ and $h \geq 2$. We have $h=h\left(C_{1}\right)=\min \left\{i: 2^{m-2} \mid k^{i}\right\}$ by Lemma 3.6. It implies that

$$
r(h-1)<m-2 \leq r h .
$$

Since $h=h\left(C_{0}\right)$, by Lemma 3.5 we have

$$
k^{h-1}<m \leq k^{h} .
$$

Combining (3.1) and (3.2), we obtain

$$
2^{r(h-1)} \leq k^{h-1} \leq m-1 \leq r h+1,
$$

so $h \leq 3$ and $r \leq 2$. By an easy computation, we have $(m, k, h, r)=(5,4,2,2),(6,4,2,2),(5,2,3,1)$ or $(4,2,2,1)$.

By computations we know that both $G(16,2)$ and $G(32,4)$ are symmetric. For $G(32,2)$ and $G(64,4)$, 
by Lemmas 3.2 and 3.3, we have $\operatorname{ind}_{32}^{2}(4)=8$, and for any vertex $a$ in $C_{1}$ which has positive indegree, $\operatorname{ind}_{32}^{2}(a)=4$. Similarly $\operatorname{ind}_{64}^{4}(16)=16, \operatorname{ind}_{64}^{4}(\mathrm{a})=8$. Thus neither of them are symmetric.

Finally, from Theorem 1.1 it is clear that if $m, k$ satisfy one of $i)-v)$, then $G\left(2^{m}, k\right)$ is symmetric. Theorem 3.1 is proved.

\section{Properties of Digraphs Product}

Given two digraphs $G_{1}$ and $G_{2}$. Let $G_{1} \times G_{2}$ be the digraph whose vertices are the ordered pairs $\left(a_{1}, a_{2}\right)$, where $a_{i} \in G_{i}$ and there is a directed edge from $\left(a_{1}, a_{2}\right)$ to $\left(b_{1}, b_{2}\right)$ if there is a directed edge from $a_{i}$ to $b_{i}$ for $i=1$, 2. In [13] L. Somer and M. Krizek proved the following fact: Let $n=n_{1} n_{2}$ where $\operatorname{gcd}\left(n_{1}, n_{2}\right)=1$, then $G(n, k) \cong G\left(n_{1}, k\right) \times G\left(n_{2}, k\right)$. And the canonical isomorphism is given by $a \mapsto\left(a_{1}, a_{2}\right)$ where $a \equiv$ $a_{i}\left(\bmod n_{i}\right), i=1,2$. In general we have

$$
G(n, k) \cong G\left(p_{1}^{e_{1}}, k\right) \times G\left(p_{2}^{e_{2}}, k\right) \times \cdots \times G\left(p_{r}^{e_{r}}, k\right),
$$

if $n=\prod_{i=1}^{r} p_{i}^{e_{i}}$ is the prime factorization of $n$. We need this fact and the following lemma.

Lemma 4.1 ([4] Lemma 3.1) Let $n=n_{1} n_{2}$ where $\operatorname{gcd}\left(n_{1}, n_{2}\right)=1$. Let $C_{i}$ be a component of $G\left(n_{i}, k\right)$. And the cycle length of $C_{i}$ is $t_{i}$. Then $C_{1} \times C_{2}$ is a subdigraph of $G(n, k)$ consisting of $\operatorname{gcd}\left(t_{1}, t_{2}\right)$ components, each having cycles of length $\operatorname{lcm}\left(t_{1}, t_{2}\right)$.

Lemma 4.2 Let $n=n_{1} n_{2}$ where $\operatorname{gcd}\left(n_{1}, n_{2}\right)=1$. If $G\left(n_{1}, k\right)$ is symmetric, then $G(n, k)$ is symmetric.

Proof. It follows immediately from Lemma 4.1 and the fact $G(n, k) \simeq G\left(n_{1}, k\right) \times G\left(n_{2}, k\right)$.

Lemma 4.3 If $G(n, k)$ is symmetric, then $G\left(n, k^{r}\right)$ is also symmetric for any $r \geq 1$.

Proof. Assume that $G(n, k)$ has $2 m$ components, say, $C_{1}, C_{2}, \cdots, C_{2 m}$, and for each $i=1,2, \cdots, m$ there exists an isomorphism $\varphi_{i}$ of digraphs:

$$
\varphi_{i}: C_{i} \rightarrow C_{i+m} \text {. }
$$

If two vertices $x, y$ are in the same component of $G\left(n, k^{r}\right)$, then there exists a vertex $z$ and positive integers $u, v$ and $x^{k^{u}} \equiv z(\bmod n), \quad y^{k^{v}} \equiv z(\bmod n)$ which implies that $x, y$ are in the same component of $G(n, k)$. It follows that if $D$ is a component of $G\left(n, k^{r}\right)$, then there exists a $j \in\{1,2, \cdots, 2 m\}$ such that $D \subseteq C_{j}$.

Let $C_{1}=\bigcup_{i=1}^{s_{1}} D_{j}$ and $C_{m+1}=\bigcup_{i=1}^{s_{2}} E_{j}$ where $D_{j}$, $j=1,2, \cdots, s_{1}$ and $E_{l}, \quad l=1,2, \cdots, s_{2}$ are components of $G\left(n, k^{r}\right)$. If $x, y \in C_{1}$ and $x^{k^{r}} \equiv y(\bmod n)$, then there exist $y_{1}, y_{2}, \cdots, y_{r}=y$ such that $x^{k} \equiv y_{1}(\bmod n)$, and $y_{i}^{k} \equiv y_{i+1}(\bmod n)$. So $\varphi_{1}(x)^{k} \equiv \varphi_{1}(y)(\bmod n)$ and $\varphi_{1}\left(y_{i}\right)^{k}$ $\equiv \varphi_{1}\left(y_{i+1}\right)(\bmod n)$, we get $\varphi_{1}(x)^{k^{r}} \equiv \varphi_{1}(y)(\bmod n)$ and $\varphi_{1}$ still preserves arrows if we consider $C_{1}$ and
$C_{m+1}$ as subdigraphs of $G\left(n, k^{r}\right)$

It follows that $s_{1}=s_{2}$ and $\varphi_{1}$ is still an isomorphism if we consider $C_{1}$ and $C_{m+1}$ as subdigraphs of $G\left(n, k^{r}\right)$. Hence $G\left(n, k^{r}\right)$ is also symmetric. Lemma 4.3 is proved. $\square$

Let $G$ be a digragh. Let $|G|$ denote the number of vertices in $G$, and let $M(G)=\max _{c \in G}\{$ ind $(c)\}$.

Lemma 4.4 Let $G$ and $H$ be two digraphs, and $a \in G$, $b \in H$. Then ind $((a, b))=\operatorname{ind}(a)$ ind $(b), \quad M(G \times H)=$ $M(G) M(H)$, and $|G \times H|=|G||H|$.

Proof. It follows immediately from the definitions.

The following lemma is the key lemma for the proof of the main result of this paper.

Lemma 4.5 Let $O_{m}$ denote the digraph whose set of vertices is $\left\{a=a_{0}, a_{1}, \cdots, a_{m-1}\right\}$ and there is a directed edge from $a_{i}$ to $a_{j}$ if and only if $a_{j}=a_{0}=a$. Let $G$ and $H$ be two digraphs such that all vertices in $G$ and $H$ have outdegree 1 . Then $O_{m} \times G \simeq O_{m} \times H$ if and only if $G \simeq H$.

Proof. Assume that $\varphi: O_{m} \times G \rightarrow O_{m} \times H$ is an isomorphism of digraphs. Let

$$
\begin{array}{ll}
G_{0}=\{x \in G \mid \operatorname{ind}(x)=0\}, & G_{1}=\{x \in G \mid \operatorname{ind}(x)>0\}, \\
H_{0}=\{x \in H \mid \text { ind }(x)=0\}, & H_{1}=\{x \in H \mid \text { ind }(x)>0\} .
\end{array}
$$

If $x \in G_{1}$ and ind $((a, x))=\operatorname{ind}(a)$ ind $(x)>0$, then ind $(\varphi(a, x))>0$. Let $\varphi((a, x))=\left(a_{j}, x^{\prime}\right)$, then we have $x^{\prime} \in H_{1}$ and $a_{j}=a$. Now we define a map $\varphi_{1}: G_{1} \rightarrow H_{1}$ by $\varphi_{1}(x)=x^{\prime}, \quad x \in G_{1}$. Obviously, $\varphi_{1}$ is injective.

If $y^{\prime} \in H_{1}$, then there exists a vertex $(a, y)$ of positive indegree in $O_{m} \times G$ such that $\varphi((a, y))=\left(a, y^{\prime}\right)$. Hence $\varphi_{1}(y)=y^{\prime}$ and $\varphi_{1}$ is also surjective.

Now we assume that $x, y \in G_{1}$, and there is a directed edge from $x$ to $y$. Let $\varphi_{1}(x)=x^{\prime}, \varphi_{1}(y)=y^{\prime}$, by definition we have $\varphi((a, x))=\left(a, x^{\prime}\right)$ and $\varphi((a, y))=$ $\left(a, y^{\prime}\right)$. We know that there is a directed edge from $(a, x)$ to $(a, y)$, then there is also a directed edge from $\left(a, x^{\prime}\right)$ to $\left(a, y^{\prime}\right)$ since $\varphi$ preserves arrows. So there is also a directed edge from $x^{\prime}$ and $y^{\prime}$. We showed that $\varphi_{1}$ preserves arrows.

For any $y \in G_{1}$, let

$$
\begin{aligned}
& E_{0}(y)=\left\{x \in G_{0} \mid \text { there is a directed edge from } x \text { to } y\right\}, \\
& E_{1}(y)=\left\{x \in G_{0} \mid \text { there is a directed edge from } x \text { to } y\right\},
\end{aligned}
$$
then

$$
G_{0}=\bigcup_{y \in G_{1}} E_{0}(y)
$$

And the above union is a disjoint union since each vertex has outdegree 1 . If $\varphi_{1}(y)=y^{\prime}$, by Lemma 4.4 we have

$$
\begin{aligned}
\operatorname{indeg}((a, y)) & =m\left(\left|E_{0}(y)\right|+\left|E_{1}(y)\right|\right) \\
= & \operatorname{ind}\left(\left(a, y^{\prime}\right)\right)=m\left(\left|E_{0}\left(y^{\prime}\right)\right|+\left|E_{1}\left(y^{\prime}\right)\right|\right)
\end{aligned}
$$


and $\left|E_{1}(y)\right|=\left|E_{1}\left(y^{\prime}\right)\right|$ since $\varphi_{1}$ maps $E_{1}(y)$ into $E_{1}\left(y^{\prime}\right)$. Then we also have $\left|E_{0}(y)\right|=\left|E_{0}\left(y^{\prime}\right)\right|^{1}$. Now we can define a map $\varphi_{0}$ from $G_{0}$ to $H_{0}$ such that for any $x \in E_{0}(y), \varphi_{0}(x) \in E_{0}\left(\varphi_{1}(y)\right)$.

Finally we can define $\phi: G \rightarrow H$

$$
\phi(a)=\varphi_{i}(a) \text { if } a \in G_{i},
$$

for $i=0,1$. It is easy to show that $\phi$ is bijective.

Now we prove that $\phi$ preserve arrows. Suppose $x, y \in G$ and there is a directed edge from $x$ to $y$. We only need to treat the case when $x \in G_{0}$ and $y \in G_{1}$. Let $\phi(y)=\varphi_{1}(y)=y^{\prime}$. By the construction of $\varphi_{0}$ we see that $\phi(x)=\varphi_{0}(x) \in E_{0}\left(y^{\prime}\right)$, so there is also a arrow from $\phi(x)$ to $\phi(y)$. It is easy to show that the number of directed edges of $G$ is equal to the number of directed edges of $H$. Thus $\phi$ is an isomorphism. Lemma 4.5 is proved.

\section{The Main Theorem}

To begin with, we prove the following lemma.

Lemma 5.1 Let $E$ be the component of $G(64 q, 4)$ containing the vertex 0 where $q$ is odd and $F$ be another component of $G(64 q, 4)$. Then $E$ is not isomorphic to $F$. And the similar result for $G(32 q, 2)$ is also valid.

Proof. We only prove the case for $G(64 q, 4)$, the proof for $G(32 q, 2)$ is similar and we omit the details. Assume that $q=\prod_{i=1}^{r} p_{i}^{e_{i}}$ where each $p_{i}$ is an odd prime, and $e_{i} \geq 2$ if $i \leq s, \quad e_{i}=1$ if $s<i \leq r$. Let $\epsilon=0$ or 1 , and let $C_{\epsilon}$ and $C_{\epsilon}^{i}$ the components of $G(64,4)$ and $G\left(p_{i}^{e_{i}}, 4\right)$, containing the vertex $\in$ and $i=1,2, \cdots, r$ respectively. Then

$$
E \cong C_{0} \times C_{0}^{1} \times \cdots \times C_{0}^{r} .
$$

If the cycle length of $\mathrm{F}>1$, then $F$ is not isomorphic to $E$. Suppose that the cycle length of $F$ is 1 , by Lemma 4.1

$$
F \simeq C_{\epsilon} \times F_{1} \times F_{2} \times \cdots \times F_{r},
$$

where $F_{i}$ is a component of cycle length 1 contained in $G\left(p_{i}^{e_{i}}, 4\right)$. By Lemma 3.1 we can write

$$
F \cong C_{\epsilon_{0}} \times C_{\epsilon_{1}}^{1} \times \cdots \times C_{\epsilon_{r}}^{r},
$$

where $\epsilon_{i}=0$ or 1 . By computations we know that $M\left(C_{0}\right)=16, M\left(C_{1}\right)=8$. By Lemma 3.3 there exists $u_{i} \geq 1$ such that $M\left(C_{0}^{i}\right)=p_{i}^{u_{i}}$ or $2 p_{i}^{u_{i}}$, or $4 p_{i}^{u_{i}}$ if $1 \leq$ $i \leq s, \quad M\left(C_{0}^{i}\right)=1$ if $s<i \leq r$. And by Lemma 3.2 $M\left(C_{1}^{i}\right)=\operatorname{gcd}\left(\left(p_{i}-1\right) p_{i}^{e_{i}-1}, 4\right)=2$ or 4 . for any $1 \leq i$ $\leq r$. Thus

$$
M(E)=16 \prod_{i=1}^{r} M\left(C_{0}^{i}\right)=16 \prod_{i=1}^{s} M\left(C_{0}^{i}\right),
$$

$$
M(F)=M\left(C_{\epsilon}\right) \cdot \prod_{i=1}^{r} M\left(C_{\epsilon_{i}}^{i}\right) .
$$

Now if $M(E)=M(F)$, we have $\epsilon_{1}=\epsilon_{2}=\cdots=\epsilon_{s}=0$, and if $\epsilon_{0}=0$ then all $\epsilon_{i}=0, E=F$. If $\in_{0}=1$, then $s=r-1$ and $\operatorname{gcd}\left(p_{r}-1, k\right)=2$. But in this case

$$
\begin{aligned}
& |E|=\left|C_{0}\right| \cdot \prod_{i=1}^{r} C_{0}^{i}=32 \prod_{i=1}^{r-1} p_{i}^{e_{i}-1}, \\
& |F|=\left|C_{1}\right| \cdot\left|C_{1}^{r}\right| \cdot\left(\prod_{i=1}^{r-1} C_{0}^{i}\right) .
\end{aligned}
$$

Therefore we have $M(E) \neq M(F)$ or $|E| \neq|F|, E$ is not isomorphic to $F$. Lemma 5.1 is proved. $\square$

Theorem 5.1 (Main Theorem) Let $k \geq 2$ and $n=$ $2^{m} q$, where $m \geq 1$ and $q$ is odd. Then $G(n, k)$ is symmetric if and only if $G\left(2^{m}, k\right)$ is symmetric.

Proof. By Lemma 4.2 we only need to prove the necessity. The case $m=1$ is trivial, so we may assume that $m \geq 2$. Let $C_{0}$ be the component of $G\left(2^{m}, k\right)$ containing the vertex 0 , and $C_{1}$ be the component of $G\left(2^{m}, k\right)$ containing the vertex 1 . Let $h_{0}=h\left(C_{0}\right)$ and $h_{1}=h\left(C_{1}\right)$. We claim that $2 \mid k$ and $h_{0}=h_{1}$. Otherwise we assume firstly that $k$ is odd or $h_{0}<h_{1}$. In both cases we have $G_{2}\left(2^{m}, k^{h_{0}}\right) \simeq O_{2^{m-1}}$, and if $x \in G\left(2^{m}, k^{h_{0}}\right)$ and $x \neq 0$, then $\operatorname{ind}_{2^{m}}^{k^{h_{0}}}(x)<2^{m-1}$.

By Lemma $4.3 G\left(n, k^{h_{0}}\right)$ is also symmetric and $G\left(n, k^{h_{0}}\right) \simeq G\left(2^{m}, k^{h_{0}}\right) \times G\left(q, k^{h_{0}}\right)$. Let

$$
G\left(q, k^{h_{0}}\right) \simeq \bigcup_{i=1}^{s} m_{i} H_{i},
$$

where each $H_{i}$ is a connect component such that $H_{i} \simeq H_{j}$ if and only if $i=j$, and $M\left(H_{i}\right) \leq M\left(H_{j}\right)$ for $i<j$. We can choose an $l$ such that $m_{l}$ is odd and $2 \mid m_{j}$ if $j>l$, since $G\left(q, k^{h_{0}}\right)$ is not symmetric. Then $G\left(2^{m}, k^{h_{0}}\right) \times\left(\bigcup_{i=1}^{l} m_{i} H_{i}\right)$ is also symmetric. Let $E=G_{2}\left(2^{m}, k^{h_{0}}\right) \times H_{l}$, by Lemma $4.1 E$ is a connected component of $G\left(2^{m}, k^{h_{0}}\right) \times\left(\bigcup_{i=1}^{l} m_{i} H_{i}\right)$ since $G_{2}\left(2^{m}, k^{h_{0}}\right)$ is a component of cycle length 1 . Let $F$ be another component of $G\left(2^{m}, k^{h_{0}}\right) \times\left(\bigcup_{i=1}^{l} m_{i} H_{i}\right)$. Suppose that

$$
E \simeq F \text {, }
$$

by Lemma 4.1 again $F$ is a component of $K \times H_{i}$, where $K$ is a component of $G\left(2^{m}, k^{h_{0}}\right)$ and $1 \leq i \leq l$. But we have

$$
\begin{aligned}
M(E) & =M\left(O_{2^{m-1}} \times H_{l}\right) \\
& =2^{m-1} M\left(H_{l}\right) \\
& \geq M(K) M\left(H_{i}\right) \\
& \geq M(F)
\end{aligned}
$$


where the equality holds if and only if $M(K)=2^{m-1}$ and $M\left(H_{i}\right)=M\left(H_{l}\right)$, which implies $K=G_{2}\left(2^{m}, k^{h_{0}}\right)$. But now we have $F=G_{2}\left(2^{m}, k^{h_{0}}\right) \times H_{i}$ and

$$
\mathrm{O}_{2^{m-1}} \times H_{l} \simeq \mathrm{O}_{2^{m-1}} \times H_{i} \text {. }
$$

Hence $H_{l} \simeq H_{i}$ by Lemma 4.5, $i=l$. We show that there are exactly $m_{l}$ components contained in $G\left(2^{m}, k^{h_{0}}\right)$ $\times\left(\bigcup_{i=1}^{l} m_{i} H_{i}\right)$ which are isomorphic to $E$.

It is contrary to the fact that $G\left(2^{m}, k^{h_{0}}\right) \times\left(\bigcup_{i=1}^{l} m_{i} H_{i}\right)$ is symmetric.

Now we have $2 \mid k$, if $h_{0}>h_{1}$, consider

$$
G\left(2^{m}, k^{h_{1}}\right)=G_{1}\left(2^{m}, k^{h_{1}}\right) \bigcup G_{2}\left(2^{m}, k^{h_{1}}\right) .
$$

We have $G_{1}\left(2^{m}, k^{h_{1}}\right) \simeq O_{2^{m-1}}$ and

$$
M\left(G_{2}\left(2^{m}, k^{h_{1}}\right)\right)<M\left(G_{1}\left(2^{m}, k^{h_{1}}\right)\right) .
$$

Using the same arguments we can show that $G\left(n, k^{h_{1}}\right)$ is not symmetric. Hence $h_{0}=h_{1}=h$.

If $h=1$, then for any vertex $a \in G\left(2^{m}, k\right)$, we have $a^{k} \equiv 0\left(\bmod 2^{m}\right)$ if $a$ is even and $a^{k} \equiv 1\left(\bmod 2^{m}\right)$ if $a$ is odd. It implies that $G\left(2^{m}, k\right) \simeq 2 O_{2^{m-1}}$. $G\left(2^{m}, k\right)$ is symmetric in this case.

If $h>1$, then $m \geq 3$. Assume that $2^{r} \| k$, then we have (3.1) and (3.2), by the proof of Theorem 3.1 we have $(m, k)=(5,4),(6,4),(5,2)$ or $(4,2)$. Then the proof is completed by Lemma 5.1 and Theorem 3.1.

Corollary 5.1 Let $n, k$ be two positive integers and $2^{m} \| n, m \geq 1$. Then $G(n, k)$ is symmetric if and only if $k=1$ or $k, m$ satisfy one of $(i)-(v)$ in Theorem 3.1.

\section{References}

[1] W. Carlip and M. Mincheva, "Symmetry of Iteration Digraphs,” Czechoslovak Mathematic Journal, Vol. 58, No. 1, 2008, pp. 131-145. doi:10.1007/s10587-008-0009-8
[2] G. Chartrand and L. Lesnidk, "Graphs and Digraphs (3rd Edition),” Chapman Hall, London, 1996.

[3] Wun-Seng Chou and Igor E. Shparlinski, "On the Cycle Structure of Repeated Exponentiation Modulo a Prime," Journal of Number Theory, Vol.107, No. 2, 2004, pp. 345-356. doi:10.1016/j.jnt.2004.04.005

[4] Joe Kramer-Miller, "Structural Properties of Power Digraphs Mudulo $n$,” Manuscript.

[5] M. Krizek, F. Lucas and L. Somer, "17 Lectures on the Femat Numbers, from Number Theory to Geometry," Springer, New York, 2001.

[6] C. Lucheta, E. Miller and C. Reiter, "Digraphs from Powers Modulo p," Fibonacci Quart, Vol. 34, 1996, pp. 226-239.

[7] I. Niven, H. S. Zuckerman and H. L. Montgomery, "An Introduction to the Theory of Numbers," 5th Edition, John Wiley \& Sons, New York, 1991.

[8] T. D. Rogers, "The Graph of the Square Mapping on the Prime Fields,” Discrete Mathematics, Vol. 148, No. 1-23, 1996, pp. 317-324. doi:10.1016/0012-365X(94)00250-M

[9] L. Somer and M. Krizek, “On a Connection of Number Theory with Graph Theory,” Czechoslovak Mathematic Journal, Vol. 54, No. 2, 2004, pp. 465-485. doi:10.1023/B:CMAJ.0000042385.93571.58

[10] L. Somer and M. Krizek, "Structure of Digrphs Associated with Quadratic Congruences with Composite Moduli,” Discrete Mathematics, Vol. 306, No. 18, 2006, pp. 2174-2185. doi:10.1016/j.disc.2005.12.026

[11] L. Somer and M. Krizek, "On Semiregular Digraphs of the Congruence $x^{k} \equiv y(\bmod n)$," Commentationes Mathematicae Universitatis Carolinae, Vol. 48, No. 1, 2007, pp. 41-58.

[12] L. Szalay, “A Discrete Iteration in Number Theory," BDTF Tud. KÄozl, Vol. 8, 1992, pp. 71-91.

[13] L. Somer and M. Krizek, "On Symmetric Digrphs of the Congruence $x^{k} \equiv y(\bmod n)$,” Discrete Mathematics, Vol. 309, No. 8, 2009, pp. 1999-2009. doi:10.1016/j.disc.2008.04.009

[14] B. Wilson, "Power Digraphs Modulo n," Fibonacci Quart, Vol. 36, 1998, pp. 229-239. 